\title{
The energy-momentum tensor in lattice QCD and the Equation of State
}

\author{
Mattia Dalla Brida \\ Dipartimento di Fisica, Università di Milano-Bicocca \\ and INFN, sezione di Milano-Bicocca \\ Edificio U2, Piazza della Scienza 3 \\ 20126 Milano, Italy. \\ E-mail: mattia.dallabrida@unimib.it

\section{Leonardo Giusti} \\ Dipartimento di Fisica, Università di Milano-Bicocca \\ and INFN, sezione di Milano-Bicocca \\ Edificio U2, Piazza della Scienza 3 \\ 20126 Milano, Italy. \\ E-mail: leonardo.giusti@unimib.it

\section{Michele Pepe*} \\ INFN, Sezione di Milano-Bicocca \\ Edificio U2, Piazza della Scienza 3 \\ 20126 Milano, Italy. \\ E-mail: michele.pepe@mib.infn.it
}

\begin{abstract}
We present a new theoretical and practical strategy to renormalize non-perturbatively the energymomentum tensor in lattice QCD based on the framework of shifted boundary conditions. As a preparatory step for the fully non-perturbative calculation, we apply the strategy at 1-loop order in perturbation theory determining the renormalization constants of both the gluonic and the fermionic components of the energy-momentum tensor. Using shifted boundary conditions, the entropy density of QCD is directly related to the expectation value of the space-time components of the renormalized energy-momentum tensor. We then discuss its practical implementation by numerical simulations of QCD with 3 flavours of Wilson quarks for temperatures between $2.5 \mathrm{GeV}$ and $80 \mathrm{GeV}$.
\end{abstract}

37th International Symposium on Lattice Field Theory - Lattice2019

16-22 June 2019

Wuhan, China

${ }^{*}$ Speaker. 


\section{Introduction}

The energy-momentum tensor (EMT) is a fundamental quantity of a Quantum Field Theory since it contains the currents associated to Poincaré symmetry and scale invariance. Furthermore, the expectation values of its matrix elements are physical quantities: they are related to thermal features like the pressure, the entropy density and the energy density as well as to transport coefficients. In the continuum, the EMT is a conserved quantity and it does not renormalize; the formulation of the EMT on the lattice was investigated by Caracciolo et al. [1] who showed that the breaking of translation and rotation symmetries by the lattice regularization leads to a non trivial definition of the EMT on the lattice. The $\{10\}$-dimensional symmetric representation of the EMT in the continuum reduces to the sum of the $\{6\}$, the $\{3\}$ and the $\{1\}$ representations of the hypercubic group of the lattice with finite renormalization constants that approach 1 in the continuum limit. Imposing the validity of Ward Identities associated to Poincare symmetry, they defined the renormalization constants perturbatively, computing them at 1-loop order [2].

However, it was not clear how to define non-perturbatively the renormalization constants and, consequently, the EMT itself. Using shifted boundary conditions, in [3, 4, 5] it was proposed a non-perturbative definition of the EMT for the $S U\left(N_{c}\right)$ Yang-Mills theory. In that framework one can exploit new Ward Identities that, in the case of periodic boundary conditions, become trivial identities. It is important to note that the non-perturbative definition of the renormalization constants with shifted boundary conditions provided also a practical method for their numerical computation by Monte Carlo simulations on the lattice. In [6], the renormalization constants of the EMT for the $S U$ (3) Yang-Mills theory have been computed non-perturbatively for the first time.

In these proceedings we extend the above results to the case of QCD. We consider the Wilson formulation of quarks on the lattice and the Sheikoleslami-Wohlert improved action. In the case of QCD, the EMT has gluonic and fermionic parts that mix under renormalization; in order to disentangle those two components, it is useful to introduce a twist phase for the matter fields in the temporal boundary conditions: it can also be interpreted as an imaginary baryonic chemical potential. Similar to the pure gauge case, the setup of shifted boundary conditions not only allows a non-perturbative definition of the EMT for QCD on the lattice but it also represents again a practical method for the numerical computation by Monte Carlo simulations.

In the framework of shifted boundary conditions, the expectation values of the space-time matrix elements of the EMT do not vanish and they are related by a purely geometric multiplicative factor to the entropy density of the system. That represents an alternative method to investigate the Equation of State of a Quantum Field Theory with respect to the standard technique [7] based on the measurement of the trace anomaly. Moreover, at variance with this latter method, no ultraviolet power divergence needs to be subtracted and the continuum limit of the entropy density can be attained in a very simple way. This approach has turned out to be very successful in the case of the $S U$ (3) Yang-Mills theory where it has been used for the calculation of the Equation of State with a permille accuracy up to the temperature of $230 T_{c}$, where $T_{c}$ is the critical temperature [8,9]. The same method can be used to compute the Equation of State of QCD and we discuss its implementation by numerical simulations with 3 flavours of Wilson quarks for temperatures between $2.5 \mathrm{GeV}$ and $80 \mathrm{GeV}$, i.e. the electro-weak scale. Preliminary results have been presented in [10, 11]. Finally, another approach based on the Gradient Flow [12] has been also recently explored [13, 14]. 


\section{Lattice QCD with shifted boundary conditions}

We consider a four-dimensional lattice with temporal extent $L_{0}$ and size $L$ along the three spatial directions and we set the lattice spacing $a=1$. The gauge field is represented by the link variables $U_{\mu}(x)$ and the quark and anti-quark fields are given by $\psi(x)$ and $\bar{\psi}(x)$, respectively. The QCD action is $S=S^{G}+S^{F}$ where the gluonic part, $S^{G}$, and the fermionic part, $S^{F}$, are given by

$$
S^{G}=\frac{1}{g_{0}^{2}} \sum_{x} \sum_{\mu, v=0}^{3} \operatorname{Re} \operatorname{Tr}\left[1-U_{\mu v}(x)\right], \quad S^{F}=\sum_{x} \bar{\psi}(x)\left(D+M_{0}\right) \psi(x)
$$

where $g_{0}$ is the bare coupling, $U_{\mu v}=U_{\mu}(x) U_{v}(x+\hat{\mu}) U_{\mu}^{\dagger}(x+\hat{v}) U_{v}^{\dagger}(x)$ is the plaquette field and $M_{0}$ is the bare quark mass matrix. We consider the $O(a)$-improved Wilson-Dirac operator $D=$ $D_{\mathrm{w}}+D_{\mathrm{sw}}$ given by the sum of the massless Wilson-Dirac operator, $D_{\mathrm{w}}$, and of the SheikholeslamiWohlert (SW) term, $D_{\mathrm{sw}}$ [15]. The gauge field is shifted by a spatial vector $\boldsymbol{\xi}$ when crossing the boundary in the temporal direction and it is periodic in the spatial directions

$$
U_{\mu}\left(x_{0}+L_{0}, \mathbf{x}\right)=U_{\mu}\left(x_{0}, \mathbf{x}-L_{0} \boldsymbol{\xi}\right), \quad U_{\mu}\left(x_{0}, \mathbf{x}+L \hat{k}\right)=U_{\mu}\left(x_{0}, \mathbf{x}\right) .
$$

In a similar way, the fermionic fields satisfy periodic boundary conditions in space and shifted boundary conditions in time in addition to the usual anti-periodicity

$$
\begin{aligned}
& \psi\left(x_{0}+L_{0}, \mathbf{x}\right)=-e^{i \theta} \psi\left(x_{0}, \mathbf{x}-L_{0} \boldsymbol{\xi}\right) \quad \text { and } \quad \psi\left(x_{0}, \mathbf{x}+L \hat{k}\right)=\psi\left(x_{0}, \boldsymbol{x}\right), \\
& \bar{\psi}\left(x_{0}+L_{0}, \mathbf{x}\right)=-e^{-i \theta} \bar{\psi}\left(x_{0}, \mathbf{x}-L_{0} \boldsymbol{\xi}\right) \quad \text { and } \quad \bar{\psi}\left(x_{0}, \mathbf{x}+L \hat{k}\right)=\bar{\psi}\left(x_{0}, \mathbf{x}\right),
\end{aligned}
$$

where we have included also a non-trivial phase at the temporal boundary. It is important to note that, by a redefinition of the fermionic fields, the multiplicative phase $e^{ \pm i \theta}$ can be removed from the temporal boundary conditions and it shows up as an imaginary baryonic chemical potential. The partition function of the system and the free-energy density are given by

$$
Z\left[L_{0}, L ; \boldsymbol{\xi}, \theta\right]=\int \mathscr{D} U \mathscr{D} \psi \mathscr{D} \bar{\psi} e^{-S}, \quad f\left[L_{0}, L ; \boldsymbol{\xi}, \theta\right]=-\frac{1}{L_{0} L^{3}} \ln Z\left[L_{0}, L ; \boldsymbol{\xi}, \theta\right] .
$$

The free-energy density provides a clear physical interpretation of the shift since in the thermodynamic limit it holds that $f\left(L_{0} ; \boldsymbol{\xi}, \theta\right)=f\left(L_{0} \sqrt{1+\boldsymbol{\xi}^{2}} ; \mathbf{0}, \theta\right)$ : this equation is the generalization to QCD of a similar relation holding for the Yang-Mills theory [5].

The EMT is given by the sum of a gluonic part and a fermionic one, $T_{\mu \nu}=T_{\mu \nu}^{G}+T_{\mu \nu}^{F}$, defined by

$$
\begin{gathered}
T_{\mu \nu}^{G}(x)=\frac{1}{g_{0}^{2}} F_{\mu \alpha}^{a}(x) F_{v \alpha}^{a}(x)-\delta_{\mu \nu} \mathscr{L}^{G}(x), \quad \text { with } \quad F_{\mu \nu}^{a}(x)=2 \operatorname{Tr}\left\{\widehat{F}_{\mu v}(x) T^{a}\right\} \\
T_{\mu \nu}^{F}(x)=\frac{1}{8}\left\{\bar{\psi}(x) \gamma_{\mu}\left[\stackrel{\leftrightarrow}{\nabla}_{v}^{*}+\overleftrightarrow{\nabla}_{v}\right] \psi(x)+\bar{\psi}(x) \gamma_{v}\left[\stackrel{\leftrightarrow}{\nabla}_{\mu}^{*}+\overleftrightarrow{\nabla}_{\mu}\right] \psi(x)\right\}-\frac{1}{4} \delta_{\mu v} \mathscr{L}^{F}(x), \\
\mathscr{L}^{G}(x)=\frac{1}{4 g_{0}^{2}} F_{\alpha \beta}^{a}(x) F_{\alpha \beta}^{a}(x), \quad \text { and } \quad \mathscr{L}^{F}(x)=\bar{\psi}(x)\left\{\frac{1}{4} \gamma_{\alpha}\left(\stackrel{\leftrightarrow}{\nabla}_{\alpha}^{*}+\overleftrightarrow{\nabla}_{\alpha}\right)+M_{0}\right\} \psi(x),
\end{gathered}
$$

where $\widehat{F}_{\mu v}(x)$ is the clover discretization of the field strength tensor, $T^{a}$ are the generators of the gauge group $S U\left(N_{c}\right)$ and the covariant derivatives are $\stackrel{\leftrightarrow}{\nabla}_{\mu}=\nabla_{\mu}-\overleftarrow{\nabla}_{\mu}$ and $\stackrel{\leftrightarrow}{\nabla}_{\mu}^{*}=\nabla_{\mu}^{*}-\overleftarrow{\nabla}_{\mu}^{*}$ with

$$
\nabla_{\mu} \psi(x)=U_{\mu}(x) \psi(x+\hat{\mu})-\psi(x), \quad \nabla_{\mu}^{*} \psi(x)=\psi(x)-U_{\mu}^{\dagger}(x-\hat{\mu}) \psi(x-\hat{\mu}),
$$




$$
\bar{\psi}(x) \overleftarrow{\nabla}_{\mu}=\bar{\psi}(x+\hat{\mu}) U_{\mu}^{\dagger}(x)-\bar{\psi}(x), \quad \bar{\psi}(x) \overleftarrow{\nabla}_{\mu}^{*}=\bar{\psi}(x)-\bar{\psi}(x-\hat{\mu}) U_{\mu}(x-\hat{\mu})
$$

In this context, an interesting observable is the flavour-singlet conserved lattice vector current

$$
V_{\mu}^{c}(x)=\frac{1}{2}\left[\bar{\psi}(x+\hat{\mu}) U_{\mu}^{\dagger}(x)\left(1+\gamma_{\mu}\right) \psi(x)+\bar{\psi}(x) U_{\mu}(x)\left(\gamma_{\mu}-1\right) \psi(x+\hat{\mu})\right]
$$

whose temporal component is related to the free-energy density by the following equation

$$
i\left\langle V_{\mu}^{c}(x)\right\rangle_{\boldsymbol{\xi}, \theta}=L_{0} \frac{\partial}{\partial \theta} f\left(L_{0} ; \boldsymbol{\xi}, \theta\right)
$$

where $\langle\cdot\rangle_{\boldsymbol{\xi}, \theta}$ stands for the expectation value w.r.t. the partition function of eq. (2.5).

\section{Non-perturbative renormalization of the EMT}

In this section we discuss the conditions for the non-perturbative renormalization of the EMT on the lattice. The approach is based on imposing for renormalized quantities on the lattice the validity of some equations - namely Ward Identities (WIs) - that hold in the continuum and that follow from the fact that the EMT contains the generators of the Poincare symmetry.

The trace of $T_{\mu \nu}$ is a singlet $\{1\}$ since it is invariant under the hypercubic group; the offdiagonal elements transform under the $\{6\}$ representation and the diagonal elements of the traceless EMT belong to the $\{3\}$ representation. Those three parts renormalize independently and the renormalized EMT can be written as $T_{\mu \nu}^{R}=T_{\mu \nu}^{R,\{6\}}+T_{\mu \nu}^{R,\{3\}}+T_{\mu \nu}^{R,\{1\}}$. For a given representation of the hypercubic group, the gluonic and the fermionic parts mix together and for the sextet and the triplet components we have

$$
T_{\mu \nu}^{R,\{6\}}=Z_{G}\left(g_{0}\right) T_{\mu \nu}^{G,\{6\}}+Z_{F}\left(g_{0}\right) T_{\mu \nu}^{F,\{6\}}, \quad T_{\mu \nu}^{R,\{3\}}=z_{G}\left(g_{0}\right) T_{\mu \nu}^{G,\{3\}}+z_{F}\left(g_{0}\right) T_{\mu \nu}^{F,\{3\}} .
$$

The discussion of the renormalization of the trace is postponed to a paper we have in preparation [16]. It is important to point out that, since the EMT in the continuum limit has no anomalous dimension, the renormalization constants are scale independent and they depend only on the bare coupling $g_{0}$.

The first interesting relation is a consequence of the fact that the space-time components of the EMT are the generators of translations: it relates the free-energy density with the one-point function of the EMT and it is given by

$$
\left\langle T_{0 k}^{R,\{6\}}(x)\right\rangle_{\boldsymbol{\xi}, \theta}=-\frac{\partial}{\partial \xi_{k}} f\left(L_{0} ; \boldsymbol{\xi}, \theta\right)
$$

where the symbol for the derivative w.r.t. the $k$-component of the shift $\xi$ on the r.h.s should be interpreted in terms of a discrete approximation on the lattice. Since we have to solve that equation for the two unknown renormalization constants, $Z_{G}$ and $Z_{F}$, a first option is to consider eq. (3.2) for two different values of the fermionic phase $\theta_{1}$ and $\theta_{2}$. Another possibility is to use eq. (2.12) and write

$$
i \frac{\partial}{\partial \xi_{k}}\left\langle V_{0}^{c}(x)\right\rangle_{\boldsymbol{\xi}, \theta}=-L_{0} \frac{\partial}{\partial \theta}\left\langle T_{0 k}^{R,[6]}(x)\right\rangle_{\boldsymbol{\xi}, \theta},
$$


This equation can also be expressed in integral form integrating over $\theta$. We emphasize that, since the Wilson discretization preserves the baryonic symmetry of the continuum theory, the vector current $V_{\mu}^{c}$ is exactly conserved on the lattice and it does not renormalize.

Once the renormalization constants of the sextet representation are fixed, the renormalization constants of the triplet representation can be obtained by requiring in the thermodynamic limit that

$$
\left\langle T_{0 k}^{R,\{6\}}(x)\right\rangle_{\boldsymbol{\xi}, \theta}=\frac{\xi_{k}}{1-\xi_{k}^{2}}\left\langle T_{00}^{R,\{3\}}(x)-T_{k k}^{R,\{3\}}(x)\right\rangle_{\boldsymbol{\xi}, \theta}, \quad \theta=\theta_{1}, \theta_{2}
$$

or else

$$
\left\langle T_{0 k}^{R,\{6\}}(x)\right\rangle_{\boldsymbol{\xi}, \theta}=\xi_{k}\left\langle T_{00}^{R,\{3\}}(x)-T_{j j}^{R,\{3\}}(x)\right\rangle_{\boldsymbol{\xi}, \theta}, \quad \boldsymbol{\theta}=\theta_{1}, \theta_{2}
$$

with $j \neq k$ and $\xi_{j}=0$. The above two WIs follow from applying to the EMT the Lorentz transformation that changes the rest frame to the moving frame described by the shifted boundary conditions [5]. The WIs that we have discussed above provide a non-perturbative definition of the renormalization constants of the EMT on the lattice. Interestingly, they also represent a practical method for computing those constants by Monte Carlo simulations on the lattice similar to the case of the Yang-Mills theory [6]. Work is in progress in this respect. Finally, the validity of the above proposal has been checked by a calculation at 1-loop order in perturbation theory [16].

\section{The QCD Equation of State}

The integral method is the technique usually considered for studying the Equation of State of a quantum theory and it is based on the direct measurement of the trace anomaly of the EMT [7]. The pressure, the entropy density and the energy density can then be obtained using standard thermodynamic relations. Although that technique has been effective and very successful, it requires to take into account a second temperature scale other than the temperature at which one is interested in. In fact, the operator whose expectation value gives the trace anomaly, mixes with the identity with the consequence of having ultraviolet power divergences. The difference of the trace anomaly at two different temperatures cancels those divergences but, from the numerical viewpoint, it can be a challenge since one has to perform Monte Carlo simulations with the same bare parameters at two different temperature scales. These computational difficulties have allowed to investigate the QCD Equation of State in the continuum limit only up to temperatures of about $1 \mathrm{GeV}$ and between $1 \mathrm{GeV}$ and $2 \mathrm{GeV}$ only with a quite large value of the lattice spacing [17, 18, 19]. Furthermore, those results have been obtained by considering the rooted staggered formulation of the fermionic fields on the lattice: it is a convenient choice on the numerical side but not fully satisfactory from the theoretical viewpoint.

The framework of shifted boundary conditions provides a much simpler environment to investigate the Equation of State. In that setup the primary observable that is measured in Monte Carlo simulations is the entropy density, $s(T)$ : it is directly related to the expectation value of the space-time components of the renormalized EMT by the equation

$$
\frac{s(T)}{T^{3}}=-\frac{L_{0}^{4}\left(1+\xi^{2}\right)^{3}}{\xi_{k}}\left\langle T_{0 k}^{R}(x)\right\rangle_{\boldsymbol{\xi}, 0}=-\frac{L_{0}^{4}\left(1+\boldsymbol{\xi}^{2}\right)^{3}}{\xi_{k}}\left[Z_{G}\left\langle T_{0 k}^{G,\{6\}}\right\rangle_{\boldsymbol{\xi}, 0}+Z_{F}\left\langle T_{0 k}^{F,\{6\}}\right\rangle_{\boldsymbol{\xi}, 0}\right]
$$


There are no divergences to remove and, once the renormalization constants are known, one just needs to perform Monte Carlo simulations to compute the two expectation values that appear on the r.h.s. The continuum limit is attained in a simple way just by tuning the bare parameters so to run the numerical simulations at the physical temperature of interest. Furthermore the numerical challenges that prevent the integral method to increase the temperature above $1-2 \mathrm{GeV}$ are overcome since one does not need to take into account two temperature scales at the same bare parameters. The framework of shifted boundary conditions turned out to be efficient and successful in investigating the Equation of State of the $S U$ (3) Yang-Mills theory with a permille accuracy up to very high temperatures $[8,9]$. We now discuss its application to QCD in the completely unexplored range of temperatures between $2 \mathrm{GeV}$ and $80 \mathrm{GeV}$, i.e. the electro-weak scale. We also point out that for the pure gauge theory, it turns out that high temperature perturbation theory does not represent a reliable description of the gluon plasma and it would be interesting to study what happens for QCD.

We perform numerical simulations of lattice QCD with $O(a)$-improved Wilson fermions with the standard plaquette Wilson action for the gauge sector. We consider 3 flavours of degenerate massless quarks: this choice is justified by the fact that we plan to investigate a regime of temperatures where the effects related to the quark masses can be neglected. We study the Equation of State at 8 physical temperatures $T$ equally spaced in log scale [10,11]. For each temperature we consider 4 values of the lattice spacing corresponding to $L_{0}=4,6,8$ and 10 . The system size in the spatial directions is kept fixed at $L=288$ and we consider the shift $\xi=(1,0,0)$ : thus, $L T$ ranges from 20 to 51 and finite size effects can be neglected. The numerical simulations are almost complete: only the last runs for 3 temperatures with $L_{0}=10$ are in progress. The values of the bare coupling have been determined using the results of the ALPHA collaboration for the running coupling [20, 21, 22, 23, 24].

Finally, in order to obtain the measurement for the entropy density in eq. (4.1) and to perform the extrapolation to the continuum limit we need to compute the sextet renormalization factors, $Z_{G}$ and $Z_{F}$ : this work is in progress.

Acknowledgements We acknowledge PRACE for awarding us access to MareNostrum at Barcelona Supercomputing Center (BSC), Spain (n. 2018194651). We also acknowledge support from a CINECA-INFN agreement, providing access to Marconi at CINECA, Italy.

\section{References}

[1] S. Caracciolo, G. Curci, P. Menotti and A. Pelissetto, The Energy Momentum Tensor for Lattice Gauge Theories, Annals Phys. 197 (1990) 119.

[2] S. Caracciolo, P. Menotti and A. Pelissetto, One loop analytic computation of the energy momentum tensor for lattice gauge theories, Nucl. Phys. B 375 (1992) 195.

[3] L. Giusti and H. B. Meyer, Thermal momentum distribution from path integrals with shifted boundary conditions, Phys. Rev. Lett. 106 (2011) 131601 [arXiv:1011.2727 [hep-lat]].

[4] L. Giusti and H. B. Meyer, Thermodynamic potentials from shifted boundary conditions: the scalar-field theory case, JHEP 1111 (2011) 087 [arXiv:1110.3136 [hep-lat]].

[5] L. Giusti and H. B. Meyer, Implications of Poincare symmetry for thermal field theories in finite-volume, JHEP 1301 (2013) 140 [arXiv:1211.6669 [hep-lat]]. 
[6] L. Giusti and M. Pepe, Energy-momentum tensor on the lattice: Nonperturbative renormalization in Yang-Mills theory, Phys. Rev. D 91, 114504 (2015) [arXiv:1503.07042 [hep-lat]].

[7] G. Boyd, J. Engels, F. Karsch, E. Laermann, C. Legeland, M. Lutgemeier and B. Petersson, Thermodynamics of SU(3) lattice gauge theory, Nucl. Phys. B 469 (1996) 419 [hep-lat/9602007].

[8] L. Giusti and M. Pepe, Equation of state of a relativistic theory from a moving frame, Phys. Rev. Lett. 113 (2014) 031601 [arXiv:1403.0360 [hep-lat]].

[9] L. Giusti and M. Pepe, Equation of state of the SU(3) Yang-Mills theory: A precise determination from a moving frame, Phys. Lett. B 769 (2017) 385 [arXiv:1612.00265 [hep-lat]].

[10] M. Dalla Brida, L. Giusti and M. Pepe, QCD in a moving frame: an exploratory study, EPJ Web Conf. 175 (2018) 14012 [arXiv:1710.09219 [hep-lat]].

[11] M. Dalla Brida, L. Giusti and M. Pepe, Towards a precise determination of the equation of state of QCD at high-temperature, PoS Confinement 2018 (2019) 140 [arXiv:1904.00896 [hep-lat]].

[12] M. Lüscher, Properties and uses of the Wilson flow in lattice QCD, JHEP 1008 (2010) 071 Erratum: [JHEP 1403 (2014) 092] [arXiv:1006.4518 [hep-lat]].

[13] H. Makino and H. Suzuki, Lattice energy-momentum tensor from the Yang-Mills gradient flow-inclusion of fermion fields, PTEP 2014 (2014) 063B02 Erratum: [PTEP 2015 (2015) 079202] [arXiv:1403.4772 [hep-lat]].

[14] Y. Taniguchi, S. Ejiri, R. Iwami, K. Kanaya, M. Kitazawa, H. Suzuki, T. Umeda and N. Wakabayashi, Exploring $N_{f}=2+1$ QCD thermodynamics from the gradient flow, Phys. Rev. D 96 (2017) no.1, 014509 Erratum: [Phys. Rev. D 99 (2019) no.5, 059904] [arXiv:1609.01417 [hep-lat]].

[15] B. Sheikholeslami and R. Wohlert, Improved Continuum Limit Lattice Action for QCD with Wilson Fermions, Nucl. Phys. B 259 (1985) 572

[16] M. Dalla Brida, L. Giusti and M. Pepe, in preparation.

[17] S. Borsanyi, Z. Fodor, C. Hoelbling, S. D. Katz, S. Krieg and K. K. Szabo, Full result for the QCD equation of state with 2+1 flavors, Phys. Lett. B 730 (2014) 99 [arXiv:1309.5258 [hep-lat]].

[18] A. Bazavov et al. [HotQCD Collaboration], Equation of state in ( 2+1 )-flavor QCD, Phys. Rev. D 90 (2014) 094503 [arXiv:1407.6387 [hep-lat]].

[19] A. Bazavov, P. Petreczky and J. H. Weber, Equation of State in 2+1 Flavor QCD at High Temperatures, Phys. Rev. D 97 (2018) no.1, 014510 [arXiv:1710.05024 [hep-lat]].

[20] M. Dalla Brida et al. [ALPHA Collaboration], Determination of the QCD $\Lambda$-parameter and the accuracy of perturbation theory at high energies, Phys. Rev. Lett. 117 (2016) no.18, 182001 [arXiv:1604.06193 [hep-ph]].

[21] M. Dalla Brida et al. [ALPHA Collaboration], Slow running of the Gradient Flow coupling from 200 MeV to $4 \mathrm{GeV}$ in $N_{\mathrm{f}}=3$ QCD, Phys. Rev. D 95 (2017) no.1, 014507 [arXiv:1607.06423 [hep-lat]].

[22] M. Bruno et al. [ALPHA Collaboration], QCD Coupling from a Nonperturbative Determination of the Three-Flavor $\Lambda$ Parameter, Phys. Rev. Lett. 119 (2017) no.10, 102001 [arXiv:1706.03821 [hep-lat]].

[23] M. Dalla Brida et al. [ALPHA Collaboration], A non-perturbative exploration of the high energy regime in $N_{\mathrm{f}}=3 Q C D$, Eur. Phys. J. C 78 (2018) no.5, 372 [arXiv:1803.10230 [hep-lat]].

[24] I. Campos et al. [ALPHA Collaboration], Non-perturbative quark mass renormalisation and running in $N_{f}=3 Q C D$, Eur. Phys. J. C 78 (2018) no.5, 387 [arXiv:1802.05243 [hep-lat]]. 